\title{
ACCESSIBILITY vs. USABILITY - WHERE IS THE DIVIDING LINE?
}

\author{
Sambhavi Chandrashekar \\ sambhavic@ucl.ac.uk \\ Rachel Benedyk \\ r.benedyk@ucl.ac.uk \\ UCL Interaction Centre University College London, (U.K.)
}

\begin{abstract}
Web accessibility for people with disabilities may be looked upon as the overcoming of barriers that make it difficult for them to use Web resources. Design criteria for Web usability have evolved over the past decade but guidelines for Web accessibility are still emerging. Issues such as whether accessibility guidelines also encompass the usability needs of users with disabilities are being debated. This study analyses the relationship between accessibility and usability and concludes that accessibility includes both access and use of resources by people with disabilities, which could be supported by a combination of a set of accessibility guidelines and a set of usability guidelines for them. It proposes a criterion of distinction between these two types of guidelines and tests the idea on an experimental website with a sample of users with visual disabilities.
\end{abstract}

\section{Introduction}

The Web today is a large repository of resources. Web accessibility for people with disabilities may be looked upon as the overcoming of barriers that prevent them from using these resources. Web accessibility can be improved through the use of assistive technologies in some cases by enabling access through alternative or enhanced modes. For example, people with visual disabilities can use a screen reader software program that reads out the computer screen contents using its internal speech synthesizer and the computer's sound card. However, the content on the Web has to be in a form that can be interpreted by such technologies. A study in the USA of 50 websites found that more than $50 \%$ of them were still only partly accessible or inaccessible in 1999 (Sullivan and Matson, 2000).

\section{Web Accessibility Initiative}

The World Wide Web Consortium (W3C) launched its Web Accessibility Initiative (WAI) in 1997 to develop strategies, guidelines and resources to make the Web accessible to people with disabilities. This would mean that people with visual, auditory, physical, speech, cognitive, and neurological disabilities can perceive, understand, navigate, and interact with the websites, and that they can contribute to the Web (WAI, 2005). Web Content Accessibility Guidelines (WCAG) 1.0 (Chisholm et al., 1999), are considered the current de facto standard for content accessibility on the Web. The next version, WCAG 2.0 (Caldwell et al, 2004), is published as a working draft, which is being revised periodically. This study used the draft version dated June 30, 2005. 


\section{Accessibility and Usability}

According to Coyne \& Nielsen (2001), with current Web design practices, users without disabilities experience three times higher usability than users with visual disabilities. A study in the United Kingdom of 1000 websites by Petrie et al in 2004 (Petrie, 2004) reported that $81 \%$ of the websites did not satisfy the most basic accessibility guidelines and even where they did, there were problems in the usability of the resources by people with disabilities. Leporini \& Paterno (2002) and Theofanos and Redish (2003) stated, after studies of accessibility and usability of websites with blind screen reader users, that meeting with accessibility standards does not necessarily make a website usable by people with disabilities. These studies have published usability guidelines for people with disabilities. However, none of them has analysed the distinction between accessibility and usability. This study analyses the relationship between accessibility and usability in order to characterise the guidelines required to support Web accessibility. It attempts to find a distinction between accessibility guidelines and usability guidelines for people with disabilities, to better clarify their role in Web design.

\section{Method and results}

The method adopted for the study comprised of: (1) theoretically analysing the relationship between accessibility and usability, (2) proposing a criterion for determining the relative scope of accessibility guidelines and usability guidelines in Web design for people with disabilities, (3) user testing the idea with a sample of visually disabled users and (4) relating the outcome to the support of accessibility.

\section{Theoretical Analysis}

Several possible relationships between accessibility and usability are discussed below. In the diagrams, A denotes 'Accessibility' and U denotes 'Usability'. To begin with, accessibility and usability could be considered as mutually exclusive. There is a perceived dichotomy between the two among HCI practitioners - some claiming to be accessibility professionals and some to be usability professionals. However, there is no available basis or evidence for such a division. Hence the argument that accessibility and usability are two unrelated issues (as in Fig. 1) may be considered untenable.

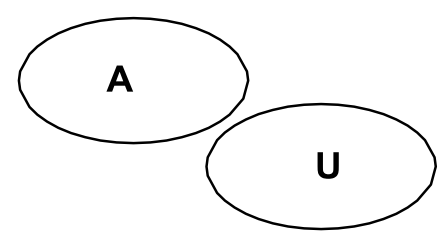

Fig. 1

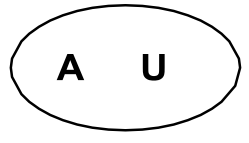

Fig. 2

There is the diametrically opposite school, which would prefer to merge accessibility with usability to form a single entity of Universal Usability (as in Fig. 2). As envisioned by Shneiderman (1998), "Universal Usability will be met when affordable, useful, and usable technology accommodates the vast majority of the global population". While this is a desirable dream, it may be difficult to achieve in practice. 
The definition of accessibility by the International Standards Organisation as "the usability of a product, service, environment or facility by people with the widest range of capabilities" [ISO TS 16071: 2003, definition 3.2] portrays accessibility as having a larger scope than usability. But literature does not support the possible conclusion derivable from this, that accessibility is a super-set of usability (as in Fig. 3). In fact, there is evidence to the contrary as can be seen below.

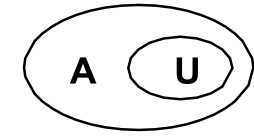

Fig 3

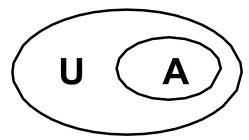

Fig. 4

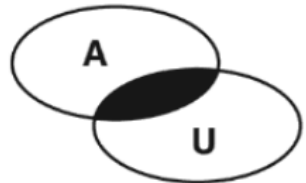

Fig 5

Henry et al (2002) consider accessibility as a subset of usability. Web usability has been popular for longer than Web accessibility and theory, techniques, and guidelines relating to Web accessibility are more recent than those of Web usability. Accessibility could thus be considered as having a narrower scope than usability (as in Fig. 4).

Having said this, it is still for consideration whether accessibility is entirely contained within usability or it merely overlaps with it. The concept of accessible Web design promoted by WAI introduced aspects into Web design that were not a regular part of usability practice and as such could be considered outside the sphere of current usability. In particular, accessibility requirements as per the WAI cover technical as well as non-technical design aspects. Usability generally focuses on non-technical user interface design issues. So accessibility does not fit neatly within usability and a relationship of overlap between the two (as in Fig. 5) appears meaningful. Boundaries defining this overlap may be dynamic and could depend on a variety of factors like nature of disability, nature of resource, content creation method, assistive technology used, user's individual capabilities, etc.

\section{Guidelines for Accessibility}

The conclusion from the above analysis is that the broad concepts of accessibility and usability overlap and that the notion of accessibility includes both a technical component and a specific usability component for people with disabilities. The fact that research-based usability guidelines for such users are being published supports this idea. This study proposes that access and use of Web resources by people with disabilities could be supported by the combination of a set of accessibility guidelines and a set of usability guidelines for them, instead of a single set of accessibility guidelines to cover the entire gamut of accessibility. It becomes necessary, however, to define the scope of accessibility guidelines unambiguously so as to distinguish them from usability guidelines that may be required specifically for people with disabilities. Accessibility measures could be seen as those that help them cross the barrier to use. Usability measures for them could be seen as those that help them use the resource more easily beyond the barrier. Based on this, the following definitions could be considered:

A guideline that recommends a design feature which enables access of underlying Web content by people with disabilities, in the absence of which design feature it is 
not technically possible for them to access that content - would be an accessibility guideline.

A guideline that recommends a design feature in whose absence the underlying content would still be technically accessible to people with disabilities while its presence would make it easier for them to use that content - would be a usability guideline for people with disabilities.

The two sets of guidelines would together work towards ensuring Web accessibility for people with disabilities.

\section{User testing}

The above idea was subjected to user testing. Six visually disabled users performed a set of six tasks on an experimental website that was designed with features in conformance/non-conformance with selected accessibility guidelines from WCAG 2.0 that applied to users with visual disabilities. Observation, think-aloud technique and pre/post-test interviews were used to gather data. Accessibility was measured in terms of success or failure of users in performing assigned tasks designed to test their ability to access content. Usability was recorded qualitatively in terms of their expressed opinions about the ease of use of the content accessed. The results of these exploratory user tests showed that non-conformance with the selected accessibility guidelines resulted in inability to access underlying resources while conformance resulted in access. It also showed that the usability of tables and forms varied based on the extent of application of usability guidelines (although this was not provable empirically).

\section{Discussion}

Barriers to access are relative and subjective; they are non-uniform across people and environments. It is difficult to determine which accessibility measures will remove the barrier to Web access for a user with a particular disability and which may merely make access and use easier. The 'user' here is actually a combination of the person and the assistive technology used. With the same disability, say visual impairment, the measures required for overcoming the accessibility barrier for a screen reader user may not apply equally for a Braille output user who is not listening to the audio output.

However, some barriers, like those in respect of perception of content and operation of interface controls, can be viewed in relation to modes of computer operation. It is possible to map perception of content with the output modes of the computer, viz., visual (content seen through the monitor); audio (content heard through the speakers); and tactile (content felt through the Braille output). Again, it is possible to map operation of interface controls with the input modes of the computer, viz., visual (using mouse); audio (using microphone); and tactile (using keyboard). Barriers associated with visual impairment are the inability to perceive content through visual output mode (monitor) and the inability to operate controls using the visual input mode (mouse). Barrier associated with hearing impairment is the inability to perceive content through the audio output mode (speakers).

This also illustrates the compensating role played by assistive technologies in providing alternative or enhanced modes. A screen reader program enables a person with 
visual impairment to perceive visual content through audio output (speakers) and operate interface controls through the tactile input (keyboard). A screen magnifier program enables a person with low vision to perceive visual content through enhanced visual output.

However, the kind of discreteness associated with perception and operation does not exist with understanding of content, which represents a continuum and so better matches with the idea of usability. This prompts the suggestion that the existing content accessibility guidelines could be enhanced / regrouped to include a set of usability guidelines for people with disabilities. The above discussion does not suggest that current guidelines under the accessibility principles mentioned above are classifiable as accessibility or usability guidelines. Rather, it is to say that a regrouping / expansion of the existing guidelines which differentiates between accessibility and usability guidelines may be more meaningful. As an example, provision of ALT Text would be an accessibility guideline; that the ALT Text should be contextually meaningful would be a usability guideline.

\section{Conclusion}

Accessibility of Web resources by people with disabilities, which involves both access and use, is best supported by a combination of a set of accessibility guidelines and a set of usability guidelines. Enhancement of the existing content accessibility guidelines into accessibility and usability guidelines for people with disabilities will make them more meaningful. This would enable better implementation of accessibility by Web developers and hopefully better use of Web resources by all.

\section{References}

Caldwell, B, Chisholm, W., Vanderheiden, G. \& White, J. (2004) "Web Content Accessibility Guidelines 2.0”, http://www.w3.org/TR/WCAG20/ (accessed 18th July 2005).

Chisholm, W., Vanderheiden, G., Jacobs, I. (eds.) (1999) Web Content Accessibility Guidelines 1.0 http://www.w3.org/TR/WCAG10/ (accessed 10th March 2005).

Coyne, K. P. and Nielsen, J., (2001) "Beyond ALT Text: Making the web easy to use for users with disabilities," Nielsen, Norman Group http://ada.ucsc.edu/ beyond_alt_text2002.pdf (accessed 25th May 2005).

Henry, S., L., et al (2002) Constructing Accessible Websites, Birmingham: Glasshaus.

Leporini, B., Paternò, F. (2002) Criteria for Usability of Accessible Web Sites, 7th ERCIM Workshop "User Interfaces for All”, Springer-Verlag, (2003), pp. 43-55.

Petrie, H., Hamilton, F., King, N. (2004) Tension, what tension? Website accessibility and visual design, Proceedings of the international cross-disciplinary workshop on Web accessibility, May 18, 2004, New York City, New York, USA.

Sullivan, T. and Matson, R. (2000) "Barriers to use: Usability and content accessibility on the web's most popular sites,” In Proc.of CUU'00, pp. 139-144, ACM Press. 
Shneiderman, B., (1998) Designing the User Interface: Strategies for Effective HumanComputer Interaction ( $3 r d$ ed.). Addison-Wesley Longman.

Theofanos, M.F., Redish, J.C. (2003) Interactions, [X. 6], November-December 2003, 38-51 http://www.redish.net/content/papers/InteractionsPaperAuthorsVer.pdf (accessed on 20th August 2005).

WAI (2005) http://www.w3.org/WAI/intro/accessibility.php (accessed 10th April 2005).

\section{Citation}

Chandrashekar, S. and Benedyk, R. (2006). Accessibility vs. Usability - where is the dividing line? Contemporary Ergonomics 2006, Editor Philip D Bust (publication of the Ergonomic Society Annual Conference, April 1-4, 2006) Taylor \& Francis Ltd: London, U.K., 231-235. 